\title{
High-grade glioma with anterior skull base erosion and intranasal extension: case report
}

\author{
Matthew T. Stib, BS, ${ }^{1}$ Michael Johnson, PA-C, ${ }^{2}$ Alan Siu, MD, ${ }^{2}$ M. Isabel Almira-Suarez, MD, ${ }^{3}$ \\ Zachary Litvack, MD, ${ }^{2}$ Ameet Singh, MD, ${ }^{4}$ and Jonathan H. Sherman, MD ${ }^{2}$ \\ ${ }^{1}$ George Washington University School of Medicine and Health Sciences; and Departments of ${ }^{2}$ Neurosurgery, ${ }^{3 P a t h o l o g y}$, and \\ ${ }^{4}$ Otolaryngology, George Washington University, Washington, DC
}

\begin{abstract}
The authors describe the case of a large WHO Grade III anaplastic oligoastrocytoma extending through the anterior skull base and into the right nasal cavity and sinuses. Glial neoplasms are typically confined to the intracranial compartment within the brain parenchyma and rarely extend into the nasal cavity without prior surgical or radiation therapy. This 42-year-old woman presented with progressive headaches and sinus congestion. MR imaging findings revealed a large intracranial lesion with intranasal extension. Endoscopic nasal biopsy revealed pathology consistent with an infiltrating glioma. The patient subsequently underwent a combined transcranial/endonasal endoscopic approach for resection of this lesion. Pathological diagnosis revealed a WHO Grade III oligoastrocytoma. This report reviews the mechanisms of extradural glioma extension. To the authors' knowledge, it is the second report of a high-grade glioma exhibiting nasal extension without prior surgical or radiation treatment.
\end{abstract}

https://thejns.org/doi/abs/10.3171/2016.4.JNS151724

KEY WORDS oligoastrocytoma; high-grade glioma; extradural glioma; nasal extension; endoscopic approach; transcranial approach; oncology

$\mathrm{H}$ IGH-GRADE glial neoplasms can rapidly grow within the confinement of the dura but rarely spread beyond these confines. Surgical manipulation or radiation has been suggested as the inciting event that weakens the protective dura and allows extradural extension of tumors. ${ }^{12}$ A particularly unusual occurrence is nasal extension of a high-grade glioma through the dura and skull base without prior neurosurgical intervention. ${ }^{2}$ To our knowledge, we report the second case of a high-grade glioma extending extracranially into the nasal cavity and sinus without any prior intervention.

\section{Case Report}

History and Examination

This 42-year-old woman with a 7-year history of chronic sinusitis presented with progressively worsening headaches associated with nausea but no vomiting, right-sided retroorbital and facial pressure, and nasal congestion and rhinorrhea. She reported a history of pain radiating down the right side of her face to her neck. She denied visual symptoms. Endoscopic examination revealed a mass with near-total obstruction of the right nasal passage at the level of the ostiomeatal complex. MR imaging demonstrated a large intraaxial mass in the right frontal lobe that was hyperintense on $\mathrm{T} 2$-weighted images and hypointense on T1-weighted images. It measured $7.5 \times 7.1 \times 6.8 \mathrm{~cm}$ with areas of streak-like enhancement without restricted diffusion. The mass demonstrated extension through the anterior skull base into the right ethmoid and maxillary sinuses and nasal cavity (Fig. 1A-C).

Results of the neurological examination were nonfocal and unremarkable. The differential diagnosis based on imaging findings suggested either a brain mass extending caudally or a sinonasal mass extending cranially, with equal probability. An endoscopic endonasal biopsy was performed by the otolaryngology service. Histopathological examination revealed a glial neoplasm subjacent to fibrotic and inflamed squamous mucosa with tumor cells positive for glial fibrillary acidic protein (GFAP) and vi- 

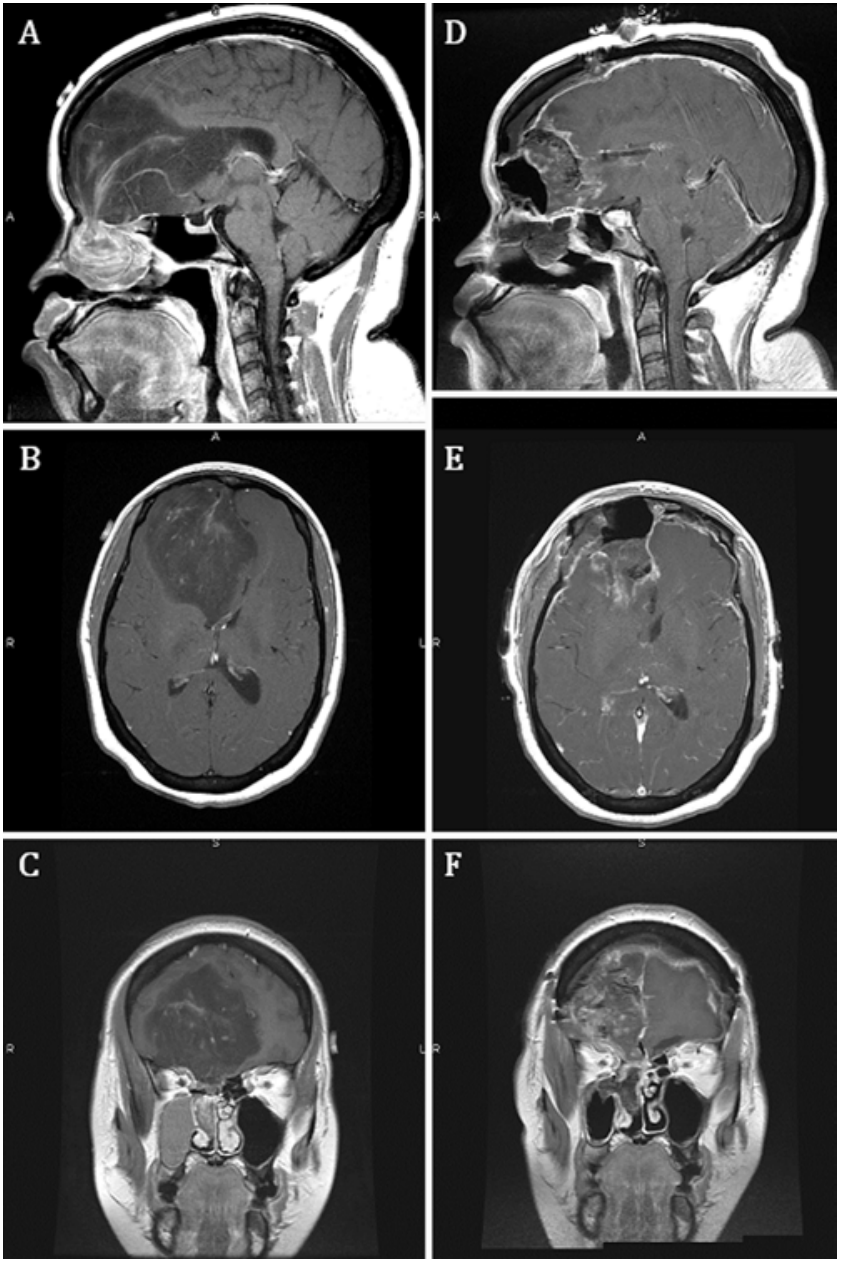

FIG. 1. Gadolinium-enhanced T1-weighted MR images. A-C: Preoperative sagittal, axial, and coronal images showing intranasal tumor extension. D-F: Postoperative sagittal, axial, and coronal images obtained after resection of tumor and repair of skull base defect.

mentin, consistent with an infiltrating glioma of unclear subtype and grade involving sinonasal tissue.

\section{Operation and Postoperative Course}

The patient underwent a simultaneous bifrontal craniotomy and endoscopic endonasal tumor resection. The endonasal approach was used to debulk the sinonasal portion of the tumor to the level of the skull base defect in the frontoethmoid region. A vascularized pedicle flap pedicled to the terminal branches of the sphenopalatine artery was harvested from the right nasal septum to aid in secondary skull base closure.

A bifrontal craniotomy was then performed, and the tumor was resected to the anterior cranial fossa floor where a chronic-appearing defect through the skull base was visualized. The bony defect was located within the fovea ethmoidalis and had smooth edges with dimensions of approximately $10 \times 7 \mathrm{~mm}$. A clear plane was identified between the rubbery tumor mass and the grossly normal adjacent neural tissue. After the tumor was removed, the anterior skull base defect was repaired using a mul-
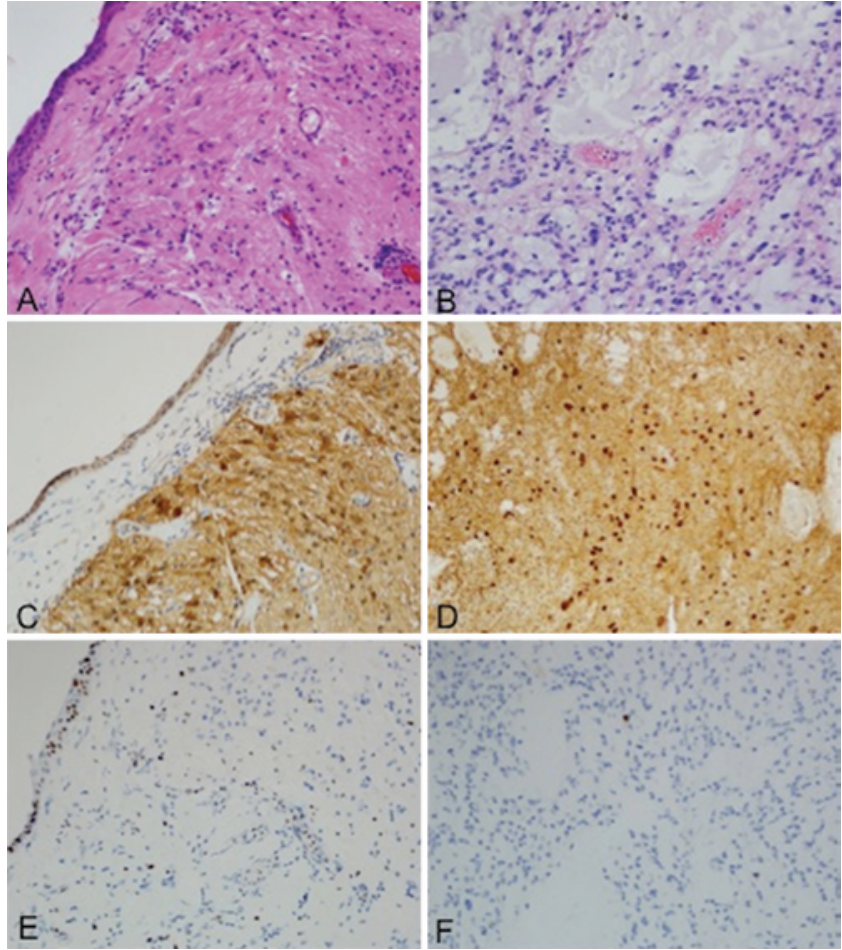

FIG. 2. Comparison between the nasal and intracranial areas of the tumor. H\&E staining of the nasal mucosa shows prominent infiltration by glial cells with gemistocytic features (A), while intraparenchymal areas of the tumor display a mixed population of gemistocytic and oligo-like cells in a microcystic background (B). The degree of pleomorphism and atypia is similar in both the nasal and the intraparenchymal areas of the tumor. IDH-1 (R132H) immunostaining shows an equivalent pattern of staining between the nasal (C) and intraparenchymal (D) portions of the tumor. The Ki-67 staining is low in both the nasal $(E)$ and intracranial $(F)$ portions of the tumor. Original magnification $\times 200$. Figure is available in color online only.

tilayered closure, with a pericranial inlay graft sutured through the defect to the nasoseptal flap to create a button-graft type closure, which was reinforced with fibrin glue. The patient tolerated the procedure well. Postoperative MR imaging confirmed adequate tumor resection and skull base reconstruction (Fig. 1D-F). The patient was discharged home without incident.

\section{Pathological Examination}

Comparison of the tumor tissue from the nasal and intracranial locations revealed similar histopathological and immunohistochemical findings (Fig. 2). The tumor was composed of 2 cell populations. The first population consisted of oval-shaped tumor cells with eccentric nuclei and eosinophilic cytoplasm resembling gemistocytes. The second population consisted of tumor cells with round, hyperchromatic nuclei resembling oligodendrocytes. The gemistocytic-like tumor cells were strongly and diffusely positive for GFAP. There was focal positivity for vimentin, highlighting the gemistocytic-like cells. Both mitotic indices and Ki-67 labeling indices were low, and only incipient microvascular proliferation was seen. Immunostaining for p53 was positive in gemistocytic areas and 
immunostaining for mutant IDH1 $(\mathrm{R} 132 \mathrm{H})$ was strongly and diffusely positive. There was no evidence of deletion of either $1 \mathrm{p}$ or $19 \mathrm{q}$ on fluorescence in situ hybridization analysis. ATRX immunohistochemistry was attempted to further classify the tumor into a more specific oligoastrocytoma versus astrocytoma differentiation; however, it was noncontributory to the diagnosis. ${ }^{13}$ MGMT methylation assay in both the nasal and intracranial portions of the tumor was pending at the time of the study. Thus, given the histopathological and immunohistochemical findings, the tumor was classified by the current WHO criteria as an anaplastic oligoastrocytoma, WHO Grade III. ${ }^{7}$

\section{Adjuvant Therapy and Follow-Up}

Follow-up endoscopy revealed a well-healed skull base reconstruction (Fig. 3). Surgery was followed by adjuvant chemotherapy with Temodar and radiation to a total of 60 Gy with conventional radiotherapy. The patient received 1 year of adjuvant Temodar on the standard 5 days on and 23 days off. MR imaging performed 16 months postoperatively showed no evidence of tumor recurrence and the patient remained neurologically intact.

\section{Discussion}

Extradural extension of primary brain tumors has been rarely reported in the literature. Prior cases of extradural extension have typically been seen in circumstances of previous neurosurgical intervention or radiation therapy. ${ }^{5,10-12,16}$ Several theories have been proposed regarding the mechanism of extracranial extension via potential dural exit points where cranial vascular and cranial nerves penetrate. Perivascular extension of tumor cells occurs mainly in the middle cranial fossa where meningeal vessels are located. ${ }^{9}$ Perineural invasion has been suggested with a description of a glioma infiltrating the dura via the trigeminal nerve. ${ }^{15}$

Sanerkin theorized that transdural extension resulted from increased intracranial pressure and herniation. ${ }^{14}$ Kawano et al. described a malignant astrocytoma with extradural extension into the frontoparietal parasagittal region. In this case, the authors proposed a novel mechanism of extension via tumor growth into arachnoid granulations and subsequent extradural spread into the adjacent sagittal sinus. ${ }^{6}$

The only previously reported case of a high-grade glioma extending into the nasal cavity in the absence of prior neurosurgical intervention was presented by Aoyama et al. ${ }^{2}$ In that case, a woman had obstructive nasal symptoms for 1 month prior to admission and changes in her cognition for nearly 1 year. The tumor was initially subtotally resected and required a subsequent operation to repair CSF rhinorrhea following radiation therapy. Our case differs from this previous case in regard to patient history, histological grade of tumor, and aggressiveness of initial surgical approach. Our patient suffered from nasal symptoms for a much longer time of 7 years and presented with a Grade III anaplastic oligoastrocytoma versus a more aggressive glioblastoma. Total resection of the tumor was performed with dissection down to the cranial floor without postoperative CSF rhinorrhea.

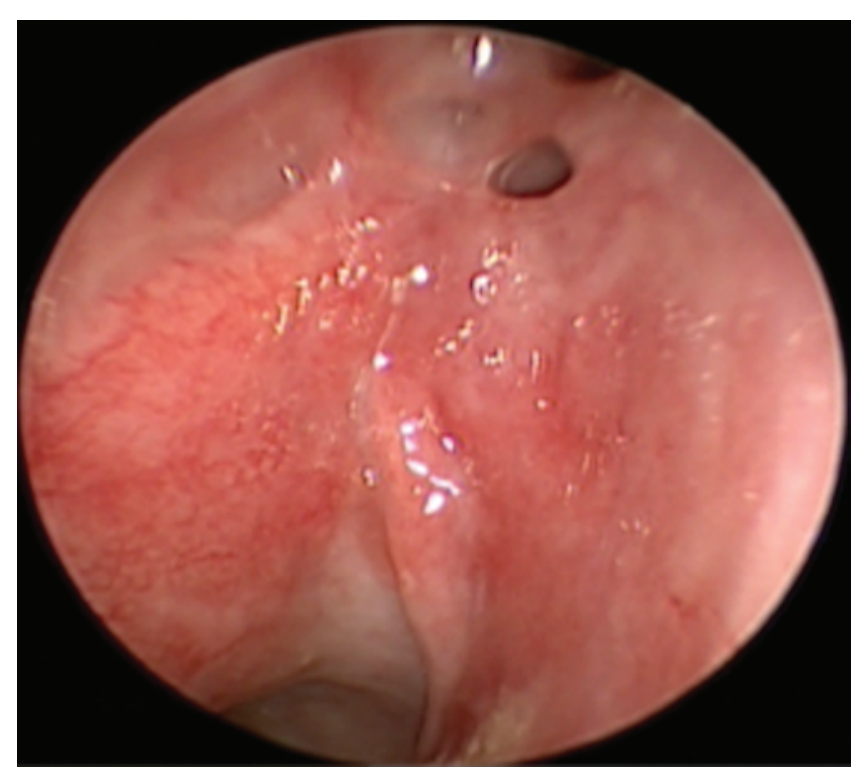

FIG. 3. Endoscopic view of a well-healed right frontoethmoidal region obtained 15 months after combined endoscopic and open resection of skull base glioma. Figure is available in color online only.

A possible mechanism for nasal extension of gliomas that has not previously been suggested includes the presence of a clinically silent congenital nasal encephalocele. Encephalocele is a herniation of neural and meningeal tissue through a congenital defect in the skull. One of the more uncommon presentations is an encephalocele penetrating into the nasal cavity. ${ }^{1}$ Typically, nasal encephaloceles present within the 1st year of life with rhinorrhea and unilateral intranasal masses on physical examination. ${ }^{3}$ However, there have been reports of adults in whom encephaloceles were never diagnosed in childhood who then presented later in life with symptoms of nasal stuffiness, frontal headaches, and rhinorrhea. ${ }^{3,8}$ In these cases, intranasal masses were discovered on physical examination that were originally diagnosed as nasal polyps. Imaging revealed bony defects within the skull base, and biopsies of the intranasal tissue revealed herniated neural and meningeal tissue.

The presentation of new sinus symptoms with intranasal CNS tissue in rare cases of adult encephalocele is similar to the presentation in our case. Our patient developed chronic sinusitis as an adult, and biopsy revealed tissue of neural origin. Also, the smooth-edged, singular and chronic appearing defect of the frontal skull base in our case suggests that the defect had been present for many years. As a possible mechanism of tumor spread in our patient, the tumor may have originated within the frontal lobe and over time insinuated itself into the neural component of the encephalocele. An alternate hypothesis is that the neural tissue within the encephalocele in the nasal cavity underwent neoplastic change and subsequently spread upward through the calvarial defect. However, considering that the bulk of the tumor was located within hemispheres, rather than the nasal cavity, this second hypothesis is less likely.

Similar to but distinct from nasal encephaloceles are 
what have been termed nasal gliomas. This terminology is not to be confused with the intranasal glioma in our case. "Nasal glioma" is a misnomer, as these tumors are generally nonneoplastic and are believed to result from erroneous embryonic development. They contain neural and/or meningeal tissues that have been isolated from the brain by cranial suture closure. ${ }^{4}$ Our case does not fit with a nasal glioma given the presence of an open skull defect and direct communication with the intracranial space.

\section{Conclusions}

This case represents the second such report of a highgrade glioma with intranasal extension without prior surgical or radiation treatment. While there exist numerous hypotheses regarding the pathway for intranasal extension, no clear mechanism has been established. The current report demonstrates that when such tumors are identified they may be effectively treated by a combined transcranial and endoscopic endonasal approach, with care taken to clearly define and primarily repair the skull base defect. While the described surgical management is not particularly novel, this case adds to the relatively small body of literature describing extradural high-grade glioma extension and suggests a novel mechanism of intranasal spread.

\section{References}

1. Anderson FM: Intranasal (sphenopharyngeal) encephalocele; a report of a case with intracranial repair and a review of the subject. Arch Otolaryngol 46:644-654, 1947

2. Aoyama I, Makita Y, Nabeshima S, Motomochi M, Masuda A: Extradural nasal and orbital extension of glioblastoma multiforme without previous surgical intervention. Surg Neurol 14:343-347, 1980

3. Blumenfeld R, Skolnik EM: Intranasal encephaloceles. Arch Otolaryngol 82:527-531, 1965

4. Gorenstein A, Kern EB, Facer GW, Laws ER Jr: Nasal gliomas. Arch Otolaryngol 106:536-540, 1980

5. Horiuchi T, Osawa M, Itoh N, Kobayashi S, Nitta J, Hongo $\mathrm{K}$ : Extradural extension of glioblastoma multiforme into the oral cavity: case report. Surg Neurol 46:42-46, 1996

6. Kawano N, Yada K, Ogawa Y, Sasaki K: Spontaneous transdural extension of malignant astrocytoma. Case report. J Neurosurg 47:766-770, 1977

7. Louis DN, Ohgaki H, Wiestler OD, Cavenee WK: WHO Classification of Tumours of the Central Nervous System. Lyon, France: World Health Organization, 2007

8. Love GL, Riehl PA: Intranasal encephalocele masking as a nasal polyp in an adult patient. Arch Otolaryngol 109:420421,1983

9. Mimata C, Itoyama Y, Kuratsu J, Uemura S, Fujioka S, Ushio Y: Anaplastic astrocytoma with extracranial extension-case report. Neurol Med Chir (Tokyo) 33:312-315, 1993

10. Orita T, Nishizaki T, Furutani Y, Aoki H: Extradural nasal and orbital extension of malignant glioma. Case report. Surg Neurol 31:395-399, 1989

11. Pompili A, Calvosa F, Caroli F, Mastrostefano R, Occhipinti E, Raus L, et al: The transdural extension of gliomas. J Neurooncol 15:67-74, 1993

12. Rubinstein LJ: Development of extracranial metastases from a malignant astrocytoma in the absence of previous craniotomy. Case report. J Neurosurg 26:542-547, 1967

13. Sahm F, Reuss D, Koelsche C, Capper D, Schittenhelm J, Heim S, et al: Farewell to oligoastrocytoma: in situ molecular genetics favor classification as either oligodendroglioma or astrocytoma. Acta Neuropathol 128:551-559, 2014

14. Sanerkin NG: Transdural spread of glioblastoma multiforme. J Pathol Bacteriol 84:228-233, 1962

15. Shenoy SN, Raja A: Spontaneous transdural spread of glioblastoma with atypical presentation. Br J Neurosurg 19:6165,2005

16. Tsutsumi S, Abe Y, Yasumoto Y, Ito M: Anaplastic pleomorphic xanthoastrocytoma with a component of anaplastic astrocytoma presenting as skull base tumor followed by downward extracranial extension. Case report. Neurol Med Chir (Tokyo) 50:1108-1112, 2010

\section{Disclosures}

The authors report no conflict of interest concerning the materials or methods used in this study or the findings specified in this paper.

\section{Author Contributions}

Conception and design: Sherman. Acquisition of data: Stib, Johnson, Almira-Suarez. Analysis and interpretation of data: Stib, Litvack, Singh. Drafting the article: Stib, Johnson, Siu, AlmiraSuarez. Critically revising the article: Sherman, Litvack, Singh. Reviewed submitted version of manuscript: all authors. Approved the final version of the manuscript on behalf of all authors: Sherman. Administrative/technical/material support: Sherman. Study supervision: Sherman.

\section{Correspondence}

Jonathan H. Sherman, Department of Neurosurgery, George Washington University Medical Center, 2150 Pennsylvania Ave. NW, Ste.7-420, Washington, DC 20037. email: jsherman0620@ gmail.com. 